\title{
POPULATION STUDY ARTICLE Head circumference at birth and intellectual disability: a nationwide cohort study
}

\author{
Kristina Aagaard ${ }^{1}$, Niels B. Matthiesen ${ }^{1,2}$, Cathrine C. Bach ${ }^{1,3}$, René T. Larsen ${ }^{1}$ and Tine B. Henriksen ${ }^{1}$
}

\begin{abstract}
BACKGROUND: Intellectual disability (ID) is a prevalent chronic disability affecting up to 1-3\% of the general population. Small head circumference at birth, a surrogate measure of foetal cerebral growth, may be a risk factor for ID. We aimed to investigate the association between the full distribution of head circumference at birth and ID.

METHODS: This cohort study was based on Danish nationwide registries and included all Danish singletons born alive from 1997 to 2013. Follow-up ended at October 2015. The data was analysed using a Cox proportional hazards regression model adjusted for a large number of potential confounders.

RESULTS: The cohort comprised 986,909 infants. Neither microcephaly nor macrocephaly at birth was consistently associated with the risk of ID. Within the normal range of head circumference, larger head circumference was associated with a decreased risk of ID (HR per standard deviation increase in head circumference $z$ score $0.85,95 \% \mathrm{Cl} 0.81-0.88$ ). The association detected within the normal range was consistent in all sensitivity analyses.
\end{abstract}

CONCLUSIONS: Intrauterine brain growth restriction may be a risk factor for ID.

Pediatric Research (2020) 87:595-601; https://doi.org/10.1038/s41390-019-0593-3

\section{INTRODUCTION}

Intellectual disability (ID) is one of society's most prevalent chronic disabilities affecting up to $1-3 \%$ of the general population. ${ }^{1}$ Various aetiological pathways leading to the development of ID have been identified. ${ }^{2}$ Nevertheless, the aetiology of ID still remains unsettled in many cases. ${ }^{2,3}$

ID is most commonly perceived as a general symptom of neurological dysfunction arising from chronic brain abnormalities. By convention, children with ID have a reduced intelligence quotient (IQ) $<70$ and concomitant deficits in adaptive functions. There are several known prenatal, perinatal and postnatal causes, of which genetic factors and intrauterine exposures are considered the most important. ${ }^{3,4}$

Foetal life is a period of rapid brain development and growth. Consequently, normal brain development crucially relies on the intrauterine environment, and various exposures during the foetal state are known to cause foetal brain growth retardation. ${ }^{5}$ Alterations in foetal brain growth may be estimated by head circumference at birth, an easily obtainable and reliable proxy for brain size. ${ }^{6-8}$ Routine measures of head circumference are performed at birth in several countries, including Denmark. ${ }^{9}$

Microcephaly during childhood has been shown to be an important predictor of ID., ${ }^{5,10}$ Nevertheless, the association between head circumference at birth and ID remains less well understood. The association between small birth size and poorer intellectual performance measured as lower IQ is well substantiated in the existing literature. Large cohort studies have shown an association between lower birth weight, shorter birth length, and smaller head circumference at birth and poorer intellectual performance in both adolescence and young adulthood. ${ }^{11-14}$ These studies did not examine the association with ID. Foetal growth restriction and low birth weight have been associated with ID. ${ }^{15-17}$ However, only few smaller studies, with limited information on relevant confounding factors, have investigated the association between head circumference at birth and the diagnosis of ID. ${ }^{15,16}$ Consequently, larger studies with detailed information on relevant confounding factors are needed to describe the association between head circumference at birth and childhood ID in detail. This may facilitate the study of early cerebral pathology and ultimately the identification of other prenatal risk factors. Moreover, the association may be of great relevance at the individual level since a diagnosis of ID reflects not only a significantly reduced IQ but also associated deficits in practical, social, and conceptual skills. ${ }^{3}$ The long-term consequences of these deficits may be modified by early identification and support. ${ }^{3}$

In this large, nationwide cohort study with long-term follow-up, we set out to estimate the association between the full range of head circumference at birth, a surrogate measure of foetal brain growth, and the risk of ID, adjusted for a large number of known and potential confounders.

\section{METHODS}

Study cohort

We identified all Danish infants born alive between January 1, 1997, and December 31, 2013.9,18 The cohort has been described previously. ${ }^{18}$ Linkage of the cohort to individual-level information from nationwide Danish registries was enabled by the unique personal identification numbers introduced in Denmark in 1968. ${ }^{19}$ Only singleton infants with a valid personal identification number, with a gestational age at birth of 22-45 weeks and no major

\footnotetext{
${ }^{1}$ Perinatal Epidemiology Research Unit \& Department of Pediatrics and Adolescent Medicine, Aarhus University Hospital, Aarhus, Denmark; ${ }^{2}$ Department of Pediatrics and Adolescent Medicine, Randers Regional Hospital, Randers, Denmark and ${ }^{3}$ Department of Pediatrics and Adolescent Medicine, Viborg Regional Hospital, Viborg, Denmark Correspondence: Kristina Aagaard (kristina.aagaard@outlook.com)
}

Received: 2 May 2019 Revised: 11 September 2019 Accepted: 18 September 2019

Published online: 2 October 2019 
Table 1. Baseline characteristics of 801,564 infants and their mothers born in Denmark 1997-2013 according to head circumference at birth



malformations, no congenital syndromes, and no diagnoses related to intrauterine alcohol exposure were considered eligible.

The study was approved by the Danish Data Protection Agency (Journal number 2012-58-006). Individual consent is not required for registry-based studies in Denmark.

Head circumference at birth

The measurement of occipitofrontal head circumference has been mandatory in Denmark for all live births since 1997. The information is stored in the Danish Medical Birth Registry along with gestational age at birth. ${ }^{9}$ Estimation of gestational age at birth is predominantly based on ultrasound measurements. ${ }^{20}$ This information allowed the calculation of sex- and gestational agespecific head circumference $z$ scores based on the population head circumference mean and standard deviation. Head circumference values were discarded as implausible if $<-5$ or $>$ +5 standard deviations (SD). ${ }^{21}$ Implausible values of gestational age were detected according to a United States algorithm and recorded as missing or replaced with gestational ages according to the first day of the last menstrual period if available. ${ }^{22}$

Intellectual disability

Cases of ID were identified using psychiatrist-assigned diagnostic codes registered in the Danish Psychiatric Central Registry. ${ }^{23}$ Diagnostic codes were recorded according to the International Classification of Diseases, the Tenth revision (ICD-10). We included all ICD-10 codes of ID (DF70 to DF79): DF70, mild intellectual disabilities; DF71, moderate intellectual disabilities; DF72, severe intellectual disabilities; DF73, profound intellectual disabilities; DF78, other intellectual disabilities; and DF79, unspecified intellectual disabilities.

\section{Covariates}

Information on covariates was obtained from several nationwide Danish Registries. Information related to birth and pregnancy was obtained from the Danish Medical Birth Registry. ${ }^{9}$ The extracted information included child birth year, sex, birth weight, plurality, maternal parity, age at delivery, pre-pregnancy body mass index (BMI), and smoking during pregnancy. Information on maternal morbidities including psychiatric illness, diabetes mellitus, hypertension, and the use of antiepileptic drugs was obtained from the Danish National Patient Registry, ${ }^{24}$ the Danish Psychiatric Central Registry, ${ }^{23}$ and the Danish National Prescription Registry. ${ }^{25}$ As in previous studies, maternal morbidities were identified using both diagnostic codes and redeemed prescriptions of relevant medication. ${ }^{26-28}$ Parental origin was defined by information on both parental citizenship and birth place extracted from the Danish Civil Registration System. Information on date of emigration, unknown place of residence, or death was also extracted from the Danish Civil Registration System. ${ }^{19}$ Finally, we extracted information on parental educational level, family disposable income, and parental cohabitation status from registries administered by 




Fig. 1 Flow chart illustrating the number of excluded and censored infants as well as the number of infants included in the primary complete case analyses

Statistics Denmark. ${ }^{29}$ The highest achieved or ongoing education of either parent defined the educational level. Disposable income was equivalence weighted, i.e. corrected for the number of individuals in the household and corrected for inflation according to the Danish Consumer Price Index. Detailed information on the registries and covariates has been presented in a previous publication. $^{18}$

\section{Statistical analyses}

We estimated the association between head circumference at birth and ID using a multivariable Cox proportional hazards regression model with robust standard errors in order to account for dependence between siblings. We performed categorized analyses comparing children born with microcephaly and macrocephaly to children born with normocephaly. We defined microcephaly as a head circumference $z$ score < $-2 \mathrm{SD}$ and macrocephaly as a head circumference $z$ score $>$ $+2 \mathrm{SD}$. Further, we performed analyses restricted to normocephalic children (head circumference $z$ score from -2SD to $+2 S D$ ) including head circumference $z$ score as a continuous variable. Lastly, we modelled head circumference as a restricted cubic spline with pre-specified knots at head circumference $z$ score values of $-5,-2,+2$, and +5 . All individuals were followed from the time of birth until the date of the first diagnosis of ID or the time of censoring due to emigration, unknown place of residence, death, or the end of follow-up on October 1, 2015, whichever occurred first. We performed all analyses both crude and adjusted for potential confounders.

Covariates were included in the analyses based on causal directed acyclic graphs and a priori knowledge ${ }^{30}$ (Supplementary Fig. S1). The main analyses were based on individuals with complete information on all covariates and were adjusted for birth year and sex, maternal parity, age at delivery, psychiatric illness, diabetes mellitus, hypertension, use of antiepileptic drugs, and smoking during pregnancy. Moreover, the analyses were adjusted for parental origin, educational level, family disposable income (included in analyses as a restricted cubic spline), and parental cohabitation status. The categorization of the covariates is shown in Table 1.

In secondary analyses, we estimated the association between head circumference $z$ score minus birth weight $z$ score (a measure of the proportionality of the child) and ID. Owing to the wellknown association between birth weight and ID, we also performed analyses adjusted for birth weight $z$ score and small for gestational age (SGA) status (birth weight $z$ score $<-1.25 S D$ ). We estimated the association between head circumference at birth and mild ID and moderate-to-profound ID (only few children were diagnosed with severe and profound ID). We performed analyses restricted to children without autism spectrum disorders (ASD; DF84.0, DF84.1, DF84.5, DF84.8, and DF84.9) or attentiondeficit hyperactivity disorder (ADHD; DF.90.0, DF90.1, DF90.8, and DF90.9) to account for the high comorbidity of ID and these disorders. Lastly, we performed analyses also adjusted for Apgar score (Apgar score categorized as $<7$ vs. $7+$ was added to the covariates from the primary analyses), a possible measure of labour and delivery complications.

We performed a number of pre-planned sensitivity analyses. In order to control for unknown or unmeasured family-related factors, we applied a within-mother between-pregnancy design using a stratified Cox regression analysis in sibling pairs of the same mother. $^{31,32}$ In order to be included in these analyses, sibling pairs were required to have discordant information on both head circumference $z$ score and the outcome. Maternal pre-pregnancy $\mathrm{BMI}$, a potential confounder, has only been recorded since 2004 and was therefore not included as a covariate in the primary analyses. Thus we carried out a sensitivity analysis restricted to cases with complete information on all variables, including BMI. In order to evaluate the effect of the applied definition of microcephaly and macrocephaly, we also performed analyses defining microcephaly as head circumference $<-3 S D$ and macrocephaly as $>+3 S D$. To account for missing data in the primary analyses as well as missing information on BMI, we performed a multiple imputation analysis under the assumption of missing at random including the entire cohort of 986,909 individuals. ${ }^{33}$ A number of variables describing foetal and maternal health, predicting the risk of missing data, were 
Table 2. The association between head circumference at birth and intellectual disability in 801,564 children born in Denmark from 1997 to 2013 Microcephaly and macrocephaly at birth and intellectual disability

\begin{tabular}{|c|c|c|c|c|c|c|c|}
\hline & \multicolumn{2}{|c|}{ No. of outcomes (\%) } & \multicolumn{2}{|c|}{ Crude HR $(95 \% \mathrm{Cl})$} & \multicolumn{2}{|c|}{ Crude HR $(95 \% \mathrm{Cl})$, complete case } & Adjusted $^{\mathrm{a}} \mathrm{HR}(95 \% \mathrm{Cl})$ \\
\hline Normocephaly (No.: 766,187$)^{\text {b }}$ & $2869(0.37)$ & & \multicolumn{2}{|l|}{1 (reference) } & \multicolumn{2}{|l|}{1 (reference) } & 1 (reference) \\
\hline Macrocephaly (No.: 19,134) ${ }^{b}$ & $63(0.33)$ & & \multicolumn{2}{|c|}{$0.75(0.59-0.94)$} & $0.79(0.61-1.01)$ & & $0.87(0.68-1.12)$ \\
\hline \multicolumn{8}{|c|}{ Head circumference $z$ score at birth and intellectual disability within normocephalic children } \\
\hline ID $2869(0.37)$ & & \multicolumn{2}{|c|}{$0.81(0.78-0.84)$} & 0.80 & $.76-0.83)$ & \multicolumn{2}{|c|}{$0.85(0.81-0.88)$} \\
\hline \multicolumn{8}{|c|}{$\begin{array}{l}\text { Children with microcephaly or macrocephaly were compared with children with normocephaly. Normocephaly: head circumference } z \text { score from }-2 S D \text { to } \\
+2 S D \text {. Microcephaly: head circumference } z \text { score }<-2 S D \text {. Macrocephaly: head circumference } z \text { score }>+2 S D \\
\text { ID intellectual disability, No. number, } C I \text { confidence interval, SD standard deviation, } H R \text { hazard ratio } \\
\text { adjusted for child birth year, child sex, parity, maternal age at delivery, maternal psychiatric illness, maternal diabetes mellitus, maternal hypertension, } \\
\text { maternal use of antiepileptic drugs, maternal smoking during pregnancy, parental origin, highest parental educational level, family disposable income, and } \\
\text { parental cohabitation status } \\
\text { b }\end{array}$} \\
\hline
\end{tabular}



Fig. 2 The association between head circumference $z$ score and ID in 801,564 children born in Denmark from 1997 to 2013 (restricted cubic spline). Restricted cubic spline with pre-specified knots at head circumference $z$ score values $-5,-2,+2$ and +5 . Adjusted for child birth year, child sex, parity, maternal age at delivery, maternal psychiatric illnes, maternal diabetes mellitus, maternal hypertension, maternal use of antiepileptic drugs, maternal smoking during pregnancy, parental origin, highest parental educational level, family disposable income, and parental cohabitation status

extracted from the Danish Medical Birth Registry and the Danish National Patient Registry and included in the imputation model in order to fulfil the assumption of missing at random (Supplementary Table S1) ${ }^{9,24}$ The number of complete cases included in the primary analyses was 801,564 (81\%). Among any given covariate excluding $\mathrm{BMI}$, the largest fraction of missing information was $14 \%$. Finally, we performed analyses stratified according to preterm birth.

Statistical analyses were performed at the server of Statistics Denmark using Stata14 (StataCorp, College Station, TX).

\section{RESULTS}

A total of 801,564 infants were included in the main analyses (Fig. 1). Baseline characteristics of the children, mothers, and parents are presented according to the presence of microcephaly, normocephaly, and macrocephaly (Table 1). Mean birth head circumference was $35.1 \mathrm{~cm}, 16,243$ children were classified as microcephalic, 766,187 as normocephalic, and 19,134 as macrocephalic. Altogether 17,455 individuals (2.2\%) were censored in the primary analyses: $2.0 \%$ emigrated, $0.2 \%$ died, and $0.03 \%$ were registered with an unknown place of residence (Fig. 1). The number of individuals diagnosed with ID was 3028 (0.38\%). The median time of follow-up was 10 years (interquartile range: 6-15 years).

Head circumference and ID

Compared to normocephaly, microcephaly was associated with an increased risk of ID (hazard ratio (HR) 1.41, 95\% confidence interval (Cl) 1.15-1.74; Table 2). Macrocephaly was not clearly associated with a decreased risk of ID (HR 0.87, 95\% Cl 0.68-1.12; Table 2). Within normocephalic children, a larger head circumference was associated with a decreased risk of ID (HR per SD increase in head circumference $z$ score $0.85,95 \% \mathrm{Cl} 0.81-0.88$; Table 2). Compared to the upper limit of normocephaly, a head circumference at the lower limit was associated with a $95 \%$ increased hazard of ID (HR 1.95, 95\% Cl 1.64-2.30). These associations were corroborated by the model using a restricted cubic spline (Fig. 2).

Secondary and sensitivity analyses

No association was found between head circumference minus birth weight $z$ score, a measure of the proportionality of the child, and ID (adjusted HR 1.02, 95\% Cl 0.98-1.06) (Supplementary Table S2). Adjusting for birth weight $z$ score or SGA did not change our results substantially within the normal range of head circumference. However, the association between microcephaly and ID was attenuated and not statistically significant (Supplementary Tables S3 and S4). Within the normal range, the association between smaller head circumference and the risk of moderate-to-profound ID was slightly stronger compared to the association between smaller head circumference and mild ID (Supplementary Table S5). Exclusion of children with ASD or ADHD did not change the associations substantially (Supplementary Table S6). The associations detected when adjusting for Apgar score were similar to the results of the primary analyses (Supplementary Table S7). The sibling analyses and the analyses adjusted for BMI confirmed the association between smaller head circumference and ID within 
Table 3. Sibling analyses: the association between head circumference at birth and intellectual disability within siblings born in Denmark from 1997 to 2013

Microcephaly and macrocephaly at birth and intellectual disability

\begin{tabular}{llll}
\hline & No. of informative sibling groups & Crude $\mathrm{HR}(95 \% \mathrm{Cl})$ & Adjusted $^{\mathrm{a}} \mathrm{HR}(95 \% \mathrm{Cl})$ \\
\hline ID & & & 1 (reference) \\
Normocephaly & 165 & $1.06(0.74-1.51)$ & $1.00(0.65-1.55)$ \\
Microcephaly & 82 & $0.97(0.58-1.61)$ & $0.85(0.49-1.46)$ \\
Macrocephaly & & &
\end{tabular}

Head circumference $z$ score at birth and intellectual disability within normocephalic children

\begin{tabular}{llll}
\hline & No. of informative sibling groups & Crude HR per SD increase $(95 \% \mathrm{Cl})$ & Adjusted $^{\mathrm{a}}$ HR per SD increase (95\% Cl) \\
\hline ID & 2452 & $0.85(0.78-0.92)$ & $0.85(0.77-0.93)$ \\
\hline
\end{tabular}

Analyses were based on sibling pairs with full information on the covariates adjusted for.

Analyses were based on sibling pairs with full information on the covariates adjusted for. Normocephaly: head circumference $z$ score from -2 SD to $+2 S D$. Microcephaly: head circumference $z$ score <-2SD. Macrocephaly: head circumference $z$ score $>+2 S D$

$I D$ intellectual disability, No. number, $H R$ hazard ratio, $S D$ standard deviation, $C l$ confidence interval

${ }^{a}$ Adjusted for child birth year, sex, parity, maternal age at delivery, and maternal smoking during pregnancy. Children with microcephaly or macrocephaly were compared with siblings with normocephaly

the normocephalic children. No association between microcephaly or macrocephaly and ID was detected in the sibling analyses (Table 3). In the analyses adjusted for BMI, the association between microcephaly and ID was attenuated and not statistically significant (Supplementary Table S8). In accordance with Fig. 2, defining microcephaly and macrocephaly at $\pm 3 S D$, the point estimates suggested stronger associations with increased risks of ID. These results were not statistically significant (Supplementary Table S9). In general, the results of the analyses based on multiple imputation including the entire cohort were similar to the results of the primary analyses (Supplementary Table S10). Compared to infants born at term, the associations between microcephaly and smaller head circumference within the normal range and ID were stronger among children born preterm (Supplementary Table S11).

\section{DISCUSSION}

This study investigated the association between the full range of head circumference at birth and ID. We found an increased risk of ID among children born with microcephaly compared to children with normocephaly. Smaller head circumference within the normal range was also associated with an increased risk of ID. In sensitivity analyses, only the association within the normal range was consistent. When comparing the two extremes of the normal range of head circumference, a head circumference at the lower extreme was associated with an almost twofold increase in the hazard of ID.

Our findings were comparable with results from previous studies investigating the association between head circumference at birth and ID, investigating the association between the percentage deviation of the observed birth head circumference from the predicted optimal birth head circumference and ID. Both studies reported associations between smaller head circumference at birth and ID. ${ }^{15,16}$

When comparing head circumference to overall birth size, measured as birth weight, no association was found between a disproportionately small head circumference relative to the overall size and ID. To our knowledge, this has not been investigated previously in newborns. However, one study showed that small head circumference relative to height in children at the age of 1 and 7 years was not more predictive for ID than small head circumference alone. ${ }^{5}$ When adjusting analyses for birth size, i.e. birth weight and SGA status, no statistically significant association was found between microcephaly at birth and ID. However, our results suggest that the association between head circumference within the normal range and ID detected in the primary analyses was not explained by overall birth size.

The degree of cognitive impairment varies significantly with the severity of $\mathrm{ID}^{2}$ and the aetiology of the disorder may also vary between categories of ID. For example, congenital syndromes are more common in children with profound as opposed to mild ID. ${ }^{34}$ Within the normal range of head circumference, we found a slightly stronger association between smaller head circumference and moderate-to-profound ID compared to mild ID. This finding is in accordance with a previous study reporting stronger associations among children with severe ID. ${ }^{15}$ In addition, a single study only found an association between head circumference at birth and severe ID but not with less severe ID. ${ }^{16}$ Our results indicate that intrauterine brain growth restriction is associated with all degrees of ID.

Previous studies have suggested aetiological distinctions between individuals with ID occurring with and without ASD or ADHD. ${ }^{35,36}$ Further, the validity of IQ tests may be impaired among children with behavioural problems and misclassification of ID may be more prevalent in children with ASD and ADHD. ${ }^{37,38}$ However, the associations between head circumference and ID remained virtually unchanged after exclusion of children with these common comorbidities. ${ }^{35,36}$

Labour and delivery complications have been associated with ID. ${ }^{15}$ Such complications may also be associated with more infant head molding and potentially confound our results. Adjusting for Apgar score, a proxy measure of complicated childbirth, revealed essentially unchanged results.

The association between microcephaly and ID disappeared in the sibling analyses and was attenuated and not statistically significant in the analyses adjusted for BMI. This finding suggests that the association shown in the primary analysis may have been due to residual confounding. Sibling analyses enable some degree of adjustment for unmeasured family-related time-stable confounders, including intrauterine, environmental, socioeconomic factors, and genetic factors. ${ }^{31}$ The association between microcephaly and ID has previously been shown to differ by socioeconomic status, and several genetic causes of microcephaly and mental retardation have been identified. ${ }^{5,39}$ To our knowledge, no 
previous studies have utilized sibling analyses to investigate the association between head circumference at birth and ID.

No statistically significant associations between micro- and macrocephaly and ID were present after changing the definition to $-3 \mathrm{SD}$ and $+3 \mathrm{SD}$. Considering the wide confidence intervals, these analyses were most likely underpowered and therefore incapable of reproducing the associations detected in the primary analyses. The point estimates suggest a stronger association between microcephaly defined as - 3SD and ID compared to microcephaly defined as -2SD. Moreover, the point estimates suggest an association between macrocephaly defined as +3SD and ID. This is in accordance with the u-shape of the restricted cubic spline, suggesting an increase in ID among children with extreme macrocephaly (Fig. 2).

Strengths of the present study included the large size of the cohort and the ability to link the cohort to detailed and complete Danish registries, which ensured virtually complete follow-up and allowed us to perform estimations with high precision, accounting for a large number of known and potential confounders. Furthermore, the size of the cohort enabled us to perform sibling analyses which, to some degree, hold the potential to account for otherwise unquantifiable family-related confounders, such as genetics. Lastly, missing data were handled by multiple imputation under a plausible assumption of missing at random in a sensitivity analysis in order to ultimately secure the validity of the primary results.

Regardless of the adjustment for several important confounders and the performance of sibling analyses, unmeasured confounding may still have affected the reported associations. Especially, the association between microcephaly and ID may have been generated by unmeasured confounding and may not be causal. On the contrary, the association detected between smaller head circumference at birth within the normal range and ID was supported by the consistency of the association in all sensitivity analyses and the apparent dose-response association visualized by the restricted cubic spline (Fig. 2). Consequently, this association may represent a causal relationship.

Analyses based on multiple imputation suggest that selection bias due to missing data did not affect the primary analyses. Selection bias due to loss to follow-up is unlikely to have caused any major bias of the results of the study considering the registrybased design and the small percentage (2.2\%) of individuals censored during follow-up.

Measurement error of head circumference at birth and misclassification of the diagnosis of ID may also have affected the results of the study. Studies have investigated routine measurements of head circumference in populations outside Denmark and found these to be reliable in general. ${ }^{40}$ Nevertheless, both intra- and inter-observer measurement variability is expected to influence the measurements. Any measurement error of head circumference is likely to be independent of a later diagnosis of ID and thus may result in non-differential measurement error, potentially attenuating the detected associations. The recording of the specific diagnosis of ID in the Danish Psychiatric Central Registry has not been validated. Nevertheless, validation studies have proven a high validity of several other mental disorders, including schizophrenia, autism, and ADHD. ${ }^{23}$ However, children with small head circumference may be subject to more attention regarding signs of ID and may be more likely to be referred for psychiatric evaluation. This may have led to differential misclassification and an overestimation of the association between microcephaly and ID. This is, however, unlikely to apply to children born with normocephaly.

In order to eliminate common causes of abnormal head circumference and ID and to secure the internal validity of the study, we excluded children born with major malformations and congenital syndromes. Thereby we excluded a large number of children with known congenital causes of ID. Consequently, the reported prevalence of ID is lower in this study compared to existing literature reporting the prevalence of ID to be $1-3 \%{ }^{1}$ Moreover, all children are not routinely IQ tested in Denmark. IQ testing is merely performed when ID or other developmental or mental difficulties are suspected. This may have led to an underestimation of the prevalence of ID. Under-diagnosis of ID is likely to be independent of head circumference at birth. Lastly, as outlined above, we excluded children with a registered diagnosis of central nervous system (CNS) malformation. However, we cannot rule out few remaining undiagnosed cases of CNS malformations within the cohort.

The current study suggests that the association between microcephaly and ID was confounded by time-stable familyrelated confounders. Within the normal range of head circumference at birth, smaller head circumference was consistently associated with ID. Consequently, isolated microcephaly at birth in itself may not be a useful marker for the risk of ID, but growth restriction of the foetal brain may be a risk factor for ID.

\section{ACKNOWLEDGEMENTS}

The Perinatal Epidemiology Research Unit, Aarhus University Hospital, Denmark provided financial support covering salaries and purchase of data.

\section{AUTHOR CONTRIBUTIONS}

All authors met the Pediatric Research authorship requirements. N.B.M. was responsible for acquisition of data. T.B.H., N.B.M., and C.C.B. were mainly responsible for the conception and design of the study. K.A. and R.T.L. were mainly responsible for data analysis. All authors contributed to the interpretation of the data. K.A. was responsible for the initial draft of the article. Finally, all authors critically revised the article for important intellectual content and approved the final version prior to submission.

\section{ADDITIONAL INFORMATION}

The online version of this article (https://doi.org/10.1038/s41390-019-0593-3) contains supplementary material, which is available to authorized users.

Competing interests: The authors declare no competing interests.

Publisher's note Springer Nature remains neutral with regard to jurisdictional claims in published maps and institutional affiliations.

\section{REFERENCES}

1. Maulik, P. K., Mascarenhas, M. N., Mathers, C. D., Dua, T. \& Saxena, S. Prevalence of intellectual disability: a meta-analysis of population-based studies. Res Dev. Disabil. 32, 419-436 (2011).

2. Katz, G. \& Lazcano-Ponce, E. Intellectual disability: definition, etiological factors, classification, diagnosis, treatment and prognosis. Salud Publica Mex. 50, s132-s141 (2008).

3. Shea, S. E. Intellectual disability (mental retardation). Pediatr. Rev. 33, 110-121 (2012). Quiz 120-121.

4. Huang, J., Zhu, T., Qu, Y. \& Mu, D. Prenatal, perinatal and neonatal risk factors for intellectual disability: a systemic review and meta-analysis. PLOS ONE 11, e0153655 (2016).

5. Dolk, $\mathrm{H}$. The predictive value of microcephaly during the first year of life for mental retardation at seven years. Dev. Med. Child Neurol. 33, 974-983 (1991).

6. Cooke, R. W., Lucas, A., Yudkin, P. L. \& Pryse-Davies, J. Head circumference as an index of brain weight in the fetus and newborn. Early Hum. Dev. 1, 145-149 (1977).

7. Cheong, J. L. et al. Head growth in preterm infants: correlation with magnetic resonance imaging and neurodevelopmental outcome. Pediatrics 121, e1534-e1540 (2008).

8. Munakata, S. et al. Gray matter volumetric MRI differences late-preterm and term infants. Brain Dev. 35, 10-16 (2013).

9. Bliddal, M., Broe, A., Pottegard, A., Olsen, J. \& Langhoff-Roos, J. The Danish Medical Birth Register. Eur. J. Epidemiol. 33, 27-36 (2018). 
10. Harris, S. R. Measuring head circumference: update on infant microcephaly. Can. Fam. Physician 61, 680-684 (2015).

11. Lundgren, E. M., Cnattingius, S., Jonsson, B. \& Tuvemo, T. Intellectual and psychological performance in males born small for gestational age with and without catch-up growth. Pediatr. Res. 50, 91-96 (2001).

12. Flensborg-Madsen, T. \& Mortensen, E. L. Birth weight and intelligence in young adulthood and midlife. Pediatrics 139, e20163161 (2017).

13. Eide, M. G., Oyen, N., Skjaerven, R. \& Bjerkedal, T. Associations of birth size, gestational age, and adult size with intellectual performance: evidence from a cohort of Norwegian men. Pediatr. Res. 62, 636-642 (2007).

14. Bergvall, N., Iliadou, A., Tuvemo, T. \& Cnattingius, S. Birth characteristics and risk of low intellectual performance in early adulthood: are the associations confounded by socioeconomic factors in adolescence or familial effects? Pediatrics 117, 714-721 (2006).

15. Langridge, A. T. et al. Maternal conditions and perinatal characteristics associated with autism spectrum disorder and intellectual disability. PLOS ONE 8, e50963 (2013).

16. Leonard, $\mathrm{H}$. et al. Relation between intrauterine growth and subsequent intellectual disability in a ten-year population cohort of children in Western Australia. Am. J. Epidemiol. 167, 103-111 (2008).

17. Bilder, D. A. et al. Prenatal and perinatal factors associated with intellectual disability. Am. J. Intellect. Dev. Disabil. 118, 156-176 (2013).

18. Aagaard, K., Bach, C. C., Henriksen, T. B., Larsen, R. T. \& Matthiesen, N. B. Head circumference at birth and childhood developmental disorders in a nationwide cohort in Denmark. Paediatr. Perinat. Epidemiol. 32, 458-466 (2018).

19. Schmidt, M., Pedersen, L. \& Sorensen, H. T. The Danish Civil Registration System as a tool in epidemiology. Eur. J. Epidemiol. 29, 541-549 (2014).

20. Jorgensen, F. S. Ultrasonography of pregnant women in Denmark 1999-2000. Description of the development since 1980-1990. Ugeskr. Laege. 165, 4409-4415 (2003).

21. Matthiesen, N. B. et al. Congenital heart defects and indices of placental and fetal growth in a nationwide study of 924422 liveborn infants. Circulation 134, 1546-1556 (2016).

22. Talge, N. M., Mudd, L. M., Sikorskii, A. \& Basso, O. United States birth weight reference corrected for implausible gestational age estimates. Pediatrics 133, 844-853 (2014)

23. Munk-Jorgensen, P. \& Mortensen, P. B. The Danish Psychiatric Central Register. Dan. Med. Bull. 44, 82-84 (1997).

24. Lynge, E., Sandegaard, J. L. \& Rebolj, M. The Danish National Patient Register. Scand. J. Public Health 39, 30-33 (2011).

25. Kildemoes, H. W., Sorensen, H. T. \& Hallas, J. The Danish National Prescription Registry. Scand. J. Public Health 39, 38-41 (2011)
26. Olesen, J. B. et al. Validation of risk stratification schemes for predicting stroke and thromboembolism in patients with atrial fibrillation: nationwide cohort study. BMJ 342, d124 (2011).

27. Molgaard-Nielsen, D., Pasternak, B. \& Hviid, A. Oral fluconazole during pregnancy and risk of birth defects. N. Engl. J. Med. 369, 2061-2062 (2013).

28. Olsen, M., Sorensen, H. T., Hjortdal, V. E., Christensen, T. D. \& Pedersen, L. Congenital heart defects and developmental and other psychiatric disorders: a Danish nationwide cohort study. Circulation 124, 1706-1712 (2011).

29. Thygesen, L. C., Daasnes, C., Thaulow, I. \& Bronnum-Hansen, H. Introduction to Danish (nationwide) registers on health and social issues: structure, access, legislation, and archiving. Scand. J. Public Health 39, 12-16 (2011).

30. Greenland, S., Pearl, J. \& Robins, J. M. Causal diagrams for epidemiologic research. Epidemiology 10, 37-48 (1999).

31. Susser, E., Eide, M. G. \& Begg, M. Invited commentary: the use of sibship studies to detect familial confounding. Am. J. Epidemiol. 172, 537-539 (2010).

32. Obel, C., et al. The risk of attention deficit hyperactivity disorder in children exposed to maternal smoking during pregnancy - a reexamination using a sibling design. J. Child Psychol. Psychiatry 57, 532-537 (2015).

33. Sterne, J. A. et al. Multiple imputation for missing data in epidemiological and clinical research: potential and pitfalls. BMJ 338, b2393 (2009).

34. Committee to Evaluate the Supplemental Security Income Disability Program for Children with Mental Disorders et al. (eds Boat, T. F. \& Wu, J. T.) Mental Disorders and Disabilities Among Low-Income Children (National Academies Press, Washington, DC, 2015)

35. Schieve, L. A., Clayton, H. B., Durkin, M. S., Wingate, M. S. \& Drews-Botsch, C. Comparison of perinatal risk factors associated with autism spectrum disorder (ASD), intellectual disability (ID), and co-occurring ASD and ID. J. Autism Dev. Disord. 45, 2361-2372 (2015).

36. Neece, C. L., Baker, B. L. \& Lee, S. S. ADHD among adolescents with intellectual disabilities: pre-pathway influences. Res. Dev. Disabil. 34, 2268-2279 (2013).

37. Jepsen, J. R., Fagerlund, B. \& Mortensen, E. L. Do attention deficits influence IQ assessment in children and adolescents with ADHD? J. Atten. Disord. 12, 551-562 (2009).

38. Bolte, S., Dziobek, I. \& Poustka, F. Brief report: The level and nature of autistic intelligence revisited. J. Autism Dev. Disord. 39, 678-682 (2009).

39. Gilmore, E. C. \& Walsh, C. A. Genetic causes of microcephaly and lessons for neuronal development. Wiley Interdiscip. Rev. Dev. Biol. 2, 461-478 (2013).

40. West, J., Manchester, B., Wright, J., Lawlor, D. A. \& Waiblinger, D. Reliability of routine clinical measurements of neonatal circumferences and research measurements of neonatal skinfold thicknesses: findings from the Born in Bradford study. Paediatr. Perinat. Epidemiol. 25, 164-171 (2011). 